\title{
A 3D User Interface for Visualizing Neuron Location in Invertebrate Ganglia
}

\author{
Jason A. Pamplin ${ }^{1}$, Ying Zhu ${ }^{1}$, Paul S. Katz ${ }^{2}$, and Rajshekhar Sunderraman ${ }^{1}$ \\ Departments of ${ }^{1}$ Computer Science and ${ }^{2}$ Biology, \\ Georgia State University, \\ Atlanta, Georgia, USA \\ jasonainternetworkflow.com \\ \{yzhu, pkatz, rsunderraman\} egsu.edu
}

\begin{abstract}
Invertebrate nervous systems serve as important models for neuroscience research because they are comprised of relatively small numbers of individually identified neurons. There is no universal means of documenting locations of individual neurons that allows variability between specimens and can be generalized to different species. We present a new technique for visualizing and documenting neuron location. First, we describe a 3D user interface that allows neuroscientists to directly mark neuron locations on a $3 \mathrm{D}$ deformable model. A new mapping scheme is proposed that specifies the location of a neuron in a common coordinate system that accommodates the individual variability in size and shape of ganglia.
\end{abstract}

\section{Introduction}

Understanding brain function depends upon identifying neuronal elements and their connections. Molluscan nervous systems have provided important models in studies of learning, memory [1] and motor pattern generation[2] because they are comprised of individually identifiable neurons. The brains of these animals contain about 600010,000 neurons clustered in ganglia. In contrast, the mammalian brain has about $10^{11}$ neurons, which fall into about 6000 classes. Therefore, the molluscan nervous system can be used as a model for developing a database of neurons and connections if the model includes a method of identifying and recording the location of each neuron's cell body.

In opisthobranch molluscs, such as Tritonia diomedea, which we are using as our model system, the cell bodies of neurons lie on or near the surface of the ganglia. Two mapping problems must be solved: (1) a 3-D UI must allow for individual shape and size variations of Tritonia ganglia so neuroscientists can mark the locations of a neuron; and (2) coordinates must be transformed to a common coordinate system, independent of the specimen geometry, such that location information can be searchable.

\section{Background}

Neuron localization is the process of assigning each neuron a coordinate so that one can recognize the same or similar neurons in different brain specimens. Neuron local- 
ization is difficult because (1) brains can be variable in shape; (2) different terms are used to describe a given brain region in different species, and the same term is used to describe different regions; and (3) boundaries of regions are sometimes ambiguously defined [3].

Many research efforts have addressed the neuron localization problem [4][5][6][7]. The resulting method can be summarized as follows: (1) A $2 \mathrm{D}$ or $3 \mathrm{D}$ brain atlas is created from brain cross section images. The most notable example is the

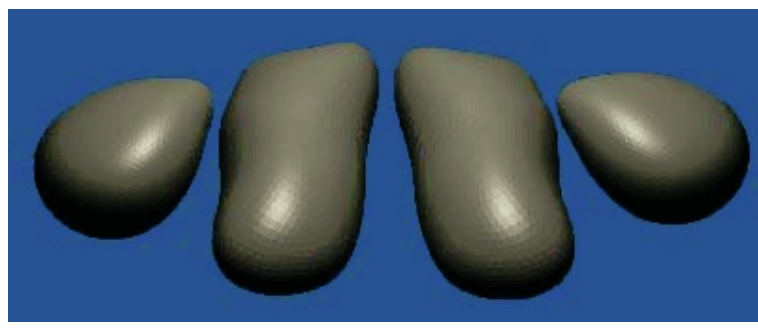

Fig. 1. Simple 3D model of Tritonia brain Talairach-Tournoux brain atlas [8]. (2) A coordinate system is defined based on certain features or landmarks on the brain atlas. (3) The atlas is then manipulated to match the features or landmarks on the given target dataset, or vice versa. The manipulations range from simple linear transformations to sophisticated physics based deformations[9][10]. (4) After the atlas is fitted to the data set, the features are assigned coordinates. This method, which depends on brain cross-section images, is used when important brain structures reside on the interior of the brain. In molluscan brains, however, neurons reside at or near the surface, allowing for a simpler approach.

\section{3D User Interface for Neuron Location Identification}

The user interface, while being easy to use, should also accurately represent neuron location relative to a brain atlas. The brain atlas is created using a standard 3D graphics package -- Blender. Figure 1 shows a reference 3D model composed of 4 individual lobes that are generated separately and then placed together to form a complete model of the Tritonia brain. Figure 2 shows a photo of a typical Tritonia brain.

The reference model must be adjusted (deformed) to visually match the actual specimen. To help the user accurately deform the model, our interface will display a photo of the specimen, overlaid with the semi-transparent 3D brain atlas. The user deforms the atlas with global scaling, rotation, and translation, to approximate the photo. Then, local deformations can fine-tune the match. A "Wire" deformation [11] algorithm is adopted here, due to its simplicity, efficiency and good interactive control. To use the atlas, the user marks a point that matches the

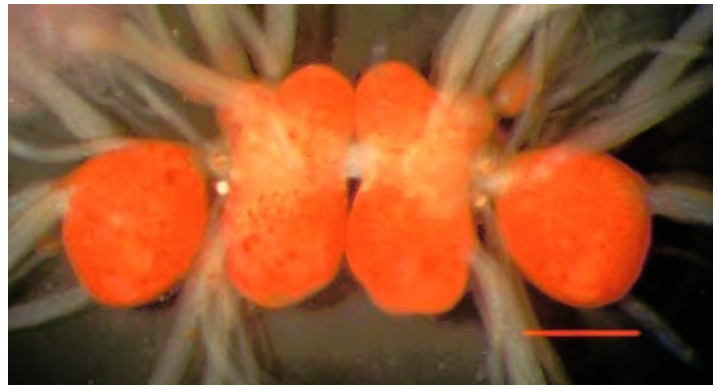

Fig. 2. Photo of typical Tritonia brain Scale bar is $0.5 \mathrm{~mm}$ 
location of the neuron under study. The location information can then be extracted by the system and stored in the database. The interface also can be used in reverse, i.e. upon selecting a location or a small region, information about neuron(s) at that location or within the region can be retrieved from the database and displayed to the user.

\section{A Common Coordinate Space for Neuron Localization}

An obvious solution to neuron localization is to keep a unique index of each vertex, record the index of the selected vertex, and then retrieve the reference $\mathrm{x}, \mathrm{y}, \mathrm{z}$ coordinates from an index table. There are several problems with this approach: (1) excessive memory is required to store an index to reference vertex table; (2) changes in the reference model that result in re-indexing will corrupt location information; and (3) storage of the reference $\mathrm{x}, \mathrm{y}, \mathrm{z}$ will make marking of a deformed model computationally intensive.

We propose mapping the $3 \mathrm{D}$ atlas to a $2 \mathrm{D}$ image space with texture mapping. Each vertex on the 3D atlas will have a corresponding texture coordinate, which is calculated with a standard parametric equation when the texture is applied to the model. Thus, each vertex on the 3D atlas is mapped to a pixel on the texture image. Once the initial correspondence is established, the texture coordinates are not recalculated during the deformation process described in section 3. Thus, for Tritonia neuron localization, the texture coordinate is stored instead of the $\mathrm{x}, \mathrm{y}, \mathrm{z}$ coordinates in 3D space.

The size and shape of the brain atlas may change, but the texture mapping remains stable, since the same parametric equation was used to map the texture coordinates for the vertices. As long as the texture is mapped completely onto the object, the relative location of each pixel of the texture image will be in the same location within a small margin of error. Thus the texture image provides a common coordinate space for comparing neurons. This technique allows for the calculation of average location for a series of observations and a statistical view of neuron location.

\section{Results and Discussion}

We have created a prototype Tritonia brain model (Figure 1) using an open source modeling tool. We are designing and developing a simple interface that allows users to mark neuron locations. The proposed solution has the following benefits: (1) changes to the reference model will not alter the location information, even if the reference vertex locations change; (2) texture coordinate calculation is performed by the modeling software with minimal computing cost; and (3) our neuron localization algorithm can be easily adapted to other species by simply creating a new 3D model.

Our solution also has it limitations: (1) this method works only for brain models where neurons are on or close to the surface; (2) users may need practice to match the atlas model to a specimen; and (3) the texture mapping may not be an exact one-toone mapping, which may lead to some inaccuracy in neuron mapping - this problem can be reduced by matching the resolution of the $2 \mathrm{D}$ image with that of the 3D atlas. 


\section{Conclusion and Future Work}

We have discussed our 3D user interface for marking neuron locations directly onto a $3 \mathrm{D}$ brain atlas. The 3D interface and the deformable 3D brain atlas can also be used to query the neuron database using positional information. We also discussed a new method for mapping neuron locations by using a technique similar to texture mapping. As a result, the user is able to compare the same neuron on different brain specimens in a common coordinate system. Although our algorithm is primarily designed for Tritonia brains, it can be easily adapted to other species by introducing a new brain atlas and adjusting the mapping equations.

\section{Acknowledgments}

Many thanks to Georgia State University colleagues: Robert Calin-Jageman, Jim Newcomb, Hao Tian, Christopher Gardner, and Lei Li; also thanks to the participants of the Identified Neuron Database Workshop (Georgia State University, December 2004). This research is funded in part by a Georgia State University Brains \& Behavior Program Seed Grant and a Georgia State University Research Initiation Grant.

\section{References}

1. Pittenger, C., Kandel, E.R., In Search of General Mechanisms for Long-lasting Plasticity: Aplysia and the hippocampus. Philosophical Transactions of Royal Society of London, Series B: Biological Science. 358(1432), (2003) 757-63

2. Getting, P.A., A Network Oscillator Underlying Swimming in Tritonia. Neuronal and Cellular Oscillators. J.W. Jacklet, Editor. Marcel Dekker, Inc., New York. (1989) 215-236

3. Bjaalie, J.G., Localization in the Brain: New Solutions Emerging. Nature Reviews: Neuroscience. 3 (2002) 322-325

4. Davatzikos, C., Spatial Normalization of 3D Brain Images Using Deformable Models. Journal of Computer Assisted Tomography, 20(4), (1996) 656-65

5. Gee J.C., Reivich, M., Bajcsy R., Elastically Deforming an Atlas to Match Anatomical Brain Images. Journal of Computer Assisted Tomography, 17(2), (2003) 225-236.

6. Roland, P.E., Zilles, K., Brain Atlases - a New Research Tool. Trends in Neuroscience, 17(11), (1994) 458-67

7. Payne B.A., Toga A.W., Surface Mapping Brain Function on 3D Models. IEEE Computer Graphics Applications, 10(5), (1990) 33-41.

8. Talairach, J., Tournoux, P., Co-planar Stereotaxic Atlas of the Human Brain. Thieme Medical Publishers, New York. (1988)

9. Toga, A.W., Brain Warping. Academic Press, New York. (1998)

10. Thompson, P., Toga, A.W. A Surface-based Technique for Warping Three-dimensional Images of the Brain. IEEE Transactions on Medical Imaging, 15(4), (1996) 402-417

11. Singh, K., Fiume, E., Wires: A Geometric Deformation Technique. Proceedings of the $25^{\text {th }}$ ACM Annual Conference on Computer Graphics and Interactive Techniques (SIGGRAPH), (1998) 405-414 